\title{
Prototipo didáctico de sistema de bombeo de agua con energía solar fotovoltaica
}

Edwin Antonio Reyes Aguilera ${ }^{1}$

\section{RESUMEN}

Con el presente estudio se pretende exponer de manera clara y ejemplificada buena parte de la información necesaria para la puesta en marcha y simulación de un sistema de bombeo autónomo con bomba sumergible alimentado por un generador solar fotovoltaico o alimentado con baterías. En el proceso de la investigación se examinaron los datos de manera científica o más específicamente en forma numérica, la investigación se realizó de manera práctica y aplicada, y consistió en, construir un prototipo de bombeo solar fotovoltaico y una parte experimental que se llevó a cabo para la validación del prototipo, utilizando para ello equipos de medición de variables meteorológicas y de medición. Este estudio se plantea a modo de guía para facilitar la enseñanza aprendizaje en los estudiantes a fin de implementar sistemas que contribuyan a mejorar tanto las condiciones de vida como la formación de la población de los países con menor nivel de desarrollo tecnológico en cuanto a materia de abastecimiento de agua, como el nuestro.

Palabras claves: Caudal, Presión, Radiación, Fotovoltaico

1 UNAN-Managua/FAREM-Estelí Correo Electróico: edwinra11@yahoo.es 


\title{
Educational prototype of a water pumping system with solar Photovoltaic energy
}

\author{
Edwin Antonio Reyes Aguilera
}

\begin{abstract}
The present study aims to give good, clear, and exemplified information necessary for the implementation and simulation of an autonomous pumping system with submersible pump powered by a photovoltaic generator or by battery. In the process of the research, data were examined scientifically or more specifically numerically, the research was conducted in a practical and applied way and consisted of building a solar photovoltaic pumping prototype, and an experimental part was carried out for the validation of the prototype, using measuring equipment and measuring meteorological variables. This study presents a guide to facilitate the teaching-learning process in students to implement systems that help improve both the lives and the formation of the population in countries with the lowest level of technological development in terms of water supply material, like ours.
\end{abstract}

Keywords: Flow, Pressure, Radiation, Photovoltaic 


\section{INTRODUCCIÓN}

La energía solar Fotovoltaica aplicada a sistemas de bombeo resulta una opción conveniente en los países de América latina, con alto nivel de radiación, para aplicaciones agrícolas y consumo humano (Vilela, 2001). Existen instalaciones para el bombeo de agua, de pequeño tamaño, en lugares aislados, instalaciones que pueden presentar diversos problemas de funcionamiento por un inadecuado dimensionado, lo cual sumado al hecho de que la energía solar disponible para los paneles Fotovoltaicos varía según el momento del día, ocasiona que el sistema trabaje fuera del punto óptimo de funcionamiento.

Fundamentados en estos inconvenientes, se hace necesario un estudio de los parámetros más importantes de dicho sistema: eficiencia del sistema, índice de radiación para el cual comienza el bombeo, las características eléctricas del generador eléctrico, el acoplamiento entre el generador y el conjunto motorbomba, las pérdidas de potencia, caudal, entre otras posibilidades. (Suerhcke, 1997).

El interés principal es promover el aprendizaje básico sobre las energías renovables, de forma manipulativa, puesto que consideramos que el aprendizaje así conseguido, es más significativo y duradero. La oportunidad de contar con una instalación que permita realizar mediciones en configuraciones distintas, evaluar y hacer mejoras en los diseños de sistemas de bombeo fotovoltaico, resulta idóneo para desarrollar un método de enseñanza que muestre las etapas que se toman en cuenta en dichos sistemas, y pueda servir además para investigaciones con el fin de entrenar estudiantes y profesionales en este campo, que aunque no es algo novedoso porque ya hay muchas investigaciones al respecto, todavía no se ha implementado un sistema que funcione para tal fin.

Con el presente estudio se pretende exponer de manera clara y ejemplificada buena parte de la información necesaria para la puesta en marcha y simulación de un sistema de bombeo autónomo con bomba sumergible alimentado por un generador solar fotovoltaico.

La Función principal de este prototipo, es la extracción de agua mediante bomba sumergible, alimentado por un generador fotovoltaico por lo cual el sistema requería de evaluación y análisis de sus parámetros de caudal, radiación, potencia del módulo solar, voltaje, intensidad, altura manométrica, presión, entre otros parámetros, para lograr una validación y de esta forma el sistema tenga una aceptación adecuada de parte de los docentes y sea de beneficio para la facultad de tener un sistema de bombeo solar fotovoltaico disponible como un medio técnico didáctico para la docencia.

\section{MATERIALES Y MÉTODOS}

\section{Instrumentos utilizados en la investigación}

1. Tanque de almacenamiento plástico de capacidad 450 litros.

2. Bomba de agua sumergible. Marca: SunPumps. Modelo: SDS - Q- 135

3. Controlador. Marca: SunPumps. Modelo: PCA30-M1

4. Reloj.

5. Panel Solar. Marca: Sharp. Modelo: NE-170U1

6. Cable TSJ 12

7. Cámara digital.

8. Vatímetro. Marca: PCE. Modelo: PA6000

9. Solarimetro. Marca: PCE. Modelo: SPM1

10. Multímetros.

11. Baterías de 12voltios, 105 Amperios

\section{Metodología de evaluación}

Pasos del proceso de validación del prototipo

\section{Parámetros de desempeño del prototipo (parámetros de validación)}

Para determinar el desempeño del prototipo se analizaran cuatro variables: Caudal de agua (Q), 
Potencia de la bomba (Watts), Tensión (Voltios) y la Irradiación Solar, (W/M2).

El análisis de un sistema de bombeo exige que se conozca el comportamiento de la bomba y del sistema de bombeo al cual pertenece. Ambos quedan caracterizados cuando se conocen las curvas de trabajo de la bomba y las pérdidas en el sistema. Las curvas se determinan en base a las condiciones de operación y requieren que se conozcan las instalaciones, estos elementos representan, a través de los gráficos, el desempeño de la bomba y del sistema y permiten determinar el funcionamiento de la bomba bajo determinadas condiciones, y los cambios que se producirán al alterar la curva de carga del sistema.

Las curvas características de una bomba se obtienen con velocidades constantes, en los sistemas de bombeo fotovoltaico, las rpm y las tensiones varían con el nivel de radiación solar incidente en el plano del generador fotovoltaico. Esto impide que las velocidades permanezcan constantes y dificulta así la obtención de las curvas, pero no nos limita a no obtenerlas. Los parámetros de validación más importante son los siguientes:

1. Determinación de la relación Potencia y altura manométrica con el caudal obtenido con la bomba. Se trata de determinar las curvas que relacionan Potencia-Caudal a diferentes alturas manométrica del caso de estudio) y altura manométrica-Caudal (fijando la tensión a 24 voltios).

2. Determinación característica Irradiancia solarPotencia en bomba e Irradiancia - caudal. Estos son datos de mucha utilidad para caracterizar y validar estos sistemas y de esta manera conocer el caudal que puede proporcionar la bomba para una irradiación dada.
Se realizaron ensayos en funcionamiento con baterías y bombeo directo.

\section{Ensayos con baterías}

Determinación de la relación de la Potencia y altura manométrica con el caudal suministrado. Se determinaron las curvas que relacionan PotenciaCaudal a diferentes alturas manométricas y altura manométrica-Caudal (fijando la tensión). Para simular las diferentes alturas se hizo a través de la válvula, en este montaje es importante señalar que la bomba fue alimentada a una tensión CC cuyo es el proporcionado por un banco de dos baterías de 12 voltios cada una conectadas en serie para una tensión total de 24 voltios. El vatímetro (PCE - PA6000), instalado proporcionó datos de voltaje y corriente lo que resulta fácil obtener el valor de potencia, $(\mathrm{V} \times \mathrm{I})$.

\section{Ensayos en bombeo directo. Sin uso de baterías}

\section{Determinación de la relación Potencia y altura} manométrica con el caudal obtenido con la bomba. En este ensayo se determinó las curvas que relacionan Potencia-Caudal (a la altura manométrica del caso de estudio) y altura manométrica-Caudal (fijando la tensión). En este montaje es importante señalar que la bomba fue alimentada a una tensión CC cuyo valor era variable en función de la irradiación.

\section{Altura manométrica-Caudal}

Este ensayo se llevó a cabo a irradiación variable lo que garantiza una tensión en bornes de salida del controlador variable. Se obtiene una curva que relacione el caudal que la bomba es capaz de suministrar para diferentes alturas manométricas, variando la altura equivalente en metros columna de agua, utilizando para ello la válvula hasta que la presión que indique el manómetro sea la deseada.

\section{Potencia-Caudal}

Es importante conocer el caudal que nos da la bomba en función de la potencia que entregan los paneles. A pesar de que el fabricante de estos paneles facilita 
estas curvas características se va a determinar para la validación. Se medirá la potencia suministrada por los paneles a través de un vatímetro. El caudal se estimará con un caudalimetro o rotámetro.

\section{Irradiancia solar-Potencia en bomba e Irradiancia -caudal}

Otro dato útil en este tipo de sistemas es conocer la potencia que puede dar la bomba para una irradiación dada. De este modo con un golpe de vista podríamos conocer que caudal tendríamos para unas condiciones de luz determinadas. Para medir la irradiación solar se utilizó un medidor llamado Piranómetro.

\section{Comparar los resultados obtenidos con la gráfica suministrada por el fabricante (SunPumps)}

Se comprueba para conocer si ambas coinciden dentro de ciertos márgenes de error que son lógicos debido a la sencillez del sistema de medición.

\section{RESULTADOS}

Seleccionar los componentes del sistema de bombeo solar fotovoltaico en función del espacio del laboratorio de la Farem, donde se ubicara el prototipo.

El proceso de selección de los elementos que forman el sistema de bombeo solar fotovoltaico, se hizo conforme a la experiencia y conocimientos sobre el tema, a fin de construir el prototipo para que fuese usado como, medio técnico - didáctico, ubicándolo en el laboratorio de energías renovables de esta facultad.

Se decidió que la tubería fuese PVC $3 / 4$ debido a que es el diámetro de la descarga de la bomba a utilizar, los elementos o accesorios a instalar tales como válvula, manómetro, uniones maleables, el rotámetro o caudalimetro, la capacidad del tanque de almacenamiento que no es de mucha capacidad, para ocupar menos espacio, además se determina que el mismo tanque simule de sondeo o pozo y al mismo tiempo se utilice como descarga de agua, es decir el agua que se succiona con la bomba regrese al mismo tanque por lo que el objetivo de selección de componentes del sistema se cumple satisfactoriamente.

\section{Caracterizar el prototipo mediante la evaluación de los parámetros y variables, en ensayos de bombeo con baterías y bombeo directo.}

Para el cumplimiento de caracterizar el prototipo, considerado el aspecto más importante durante esta investigación se ha realizado una serie de mediciones con instrumentos de medida de fácil manejo que realmente no nos garantizan que dichos resultados tengan la fidelidad deseada, pero que son de mucha utilidad para entender el funcionamiento de este prototipo de manera general.

Los resultados más importantes que se han podido extraer de todo el proceso de medición y los ensayos para las pruebas de funcionamiento del prototipo son las siguientes:

\section{Funcionamiento con baterías:}

Se conectó la bomba a la tensión de Corriente Continua a través del banco de baterías de 12 voltios cada una conectadas en serie para una tensión total de 24 voltios.

El vatímetro que se instaló, proporcionó los datos de voltaje y corriente, que permitió realizar los cálculos de potencia en la bomba que fueron de 43.50 a 113.25 watts, a una altura de $0 \mathrm{~m}$ mínimo y 24.6 metros máximo.

Fue posible observar en el ensayo, que a medida que se aumentaba la altura manométrica, se presenta una caída de tensión, de 1.2 voltios, de 0 a 24.6 metros, lo que permitió obtener un aumento de corriente, obtener mayor potencia de la bomba y lógicamente una reducción del caudal suministrado que va de $151 / \mathrm{min}$ a $11.80 \mathrm{l} / \mathrm{m}$, aunque no se considera un aspecto negativo, ya que se acerca a las especificaciones técnicas que nos brinda el fabricante de estas bombas. 


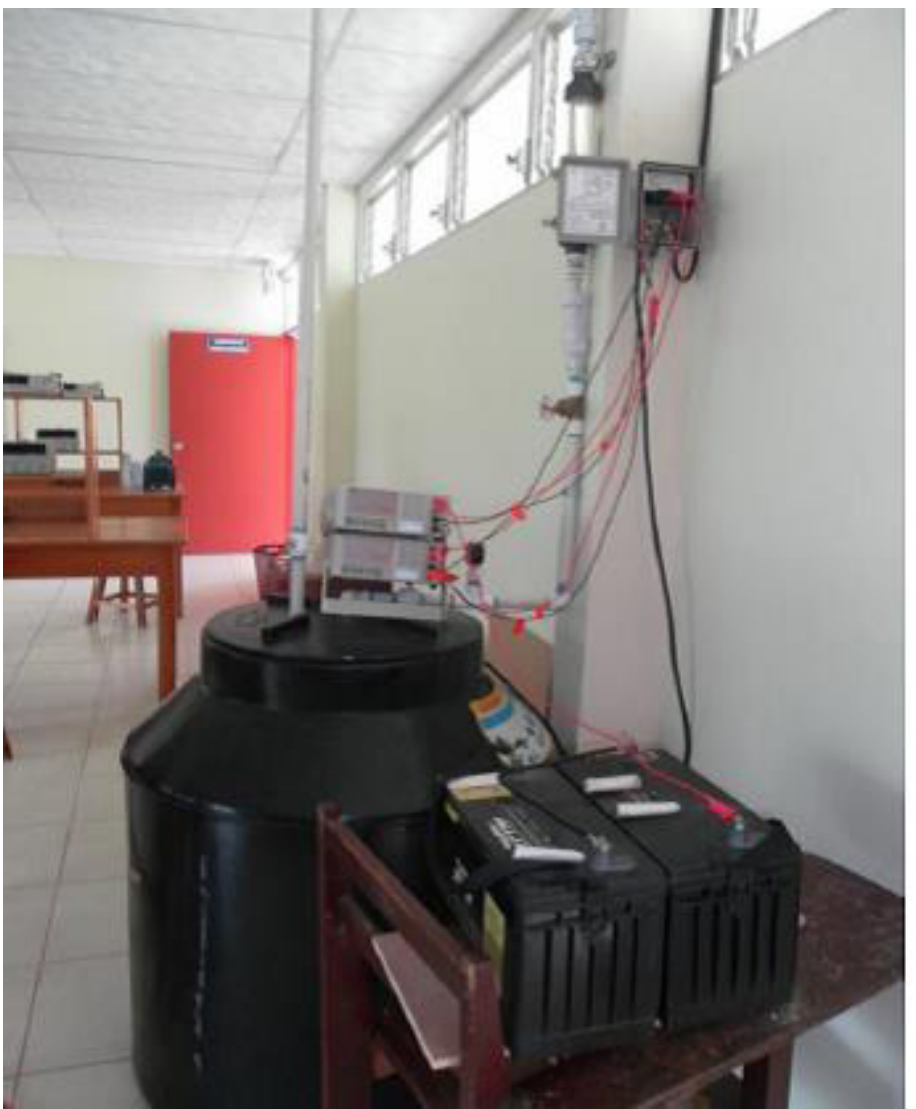

Por lo que podemos decir que a mayor altura manométrica la bomba demanda más potencia y también a mayor altura, el voltaje disminuye, aumenta la corriente y disminuye el caudal.

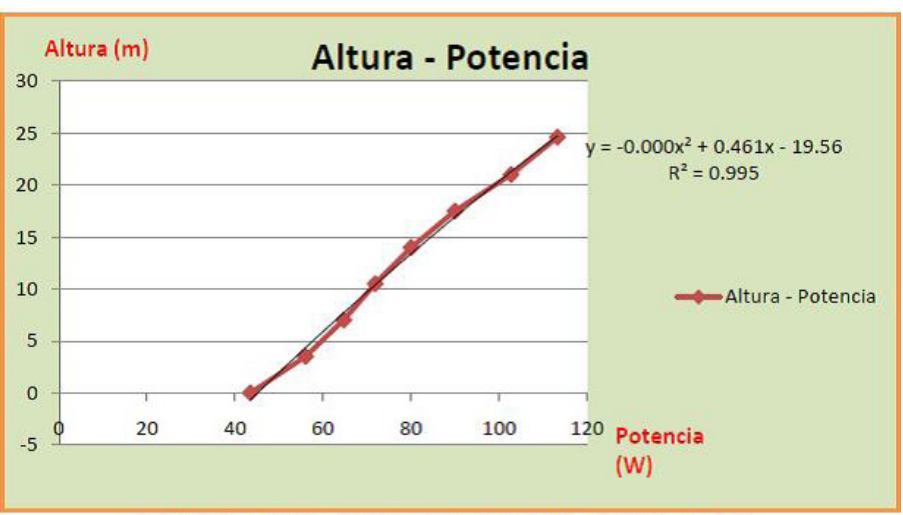

Gráfica 1: Altura para un caudal máximo de 16 l/m

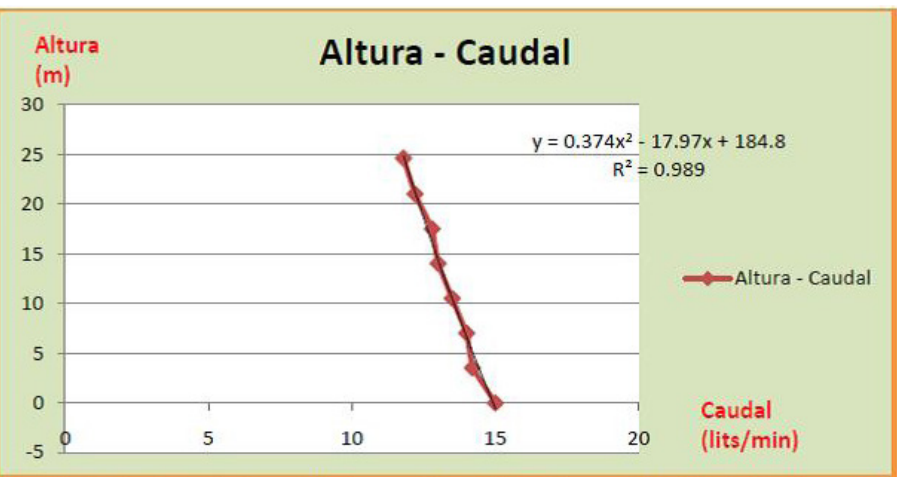

Gráfica 2: Evolución de la potencia demandada para el caudal máximo en función de la altura en bombeo

\begin{tabular}{lllllll}
\hline Medida & PSI & $\begin{array}{l}\text { Altura } \\
\text { Manometrica } \\
(\mathrm{m})\end{array}$ & $\begin{array}{l}\text { Tensión } \\
\text { Bomba } \\
(\mathrm{V})\end{array}$ & $\begin{array}{l}\text { Intensidad } \\
\text { Bomba } \\
(\mathrm{A})\end{array}$ & $\begin{array}{l}\text { Potencia } \\
\text { Motor } \\
\text { (Watts) }\end{array}$ & $\begin{array}{l}\text { Caudal } \\
\text { (lits/min) }\end{array}$ \\
\hline 1 & 0 & 0.00 & 25.00 & 1.74 & 43.50 & 15.00 \\
2 & 5 & 3.50 & 24.80 & 2.26 & 56.00 & 14.20 \\
3 & 10 & 7.00 & 24.60 & 2.63 & 64.70 & 14.00 \\
4 & 15 & 10.50 & 24.50 & 2.93 & 71.80 & 13.50 \\
5 & 20 & 14.00 & 24.3 & 3.30 & 80.00 & 13.00 \\
6 & 25 & 17.00 & 24.2 & 3.72 & 90.00 & 12.80 \\
7 & 30 & 21.00 & 24 & 4.28 & 102.70 & 12.20 \\
8 & 35 & 24.60 & 23.8 & 4.76 & 113.25 & 11.80 \\
\hline
\end{tabular}

Funcionamiento sin baterías: (Panel fotovoltaico)

Al realizar el ensayo en bombeo directo, se comprueba, que el caudal dependerá de la irradiación incidente en cada momento, lo cual hace que la potencia que suministran los paneles sea mayor, los resultados obtenidos indican que a mayor irradiación mayor será el caudal obtenido aun cuando variemos la altura a la que se desea bombear.

Al comparar los resultados obtenidos con las especificaciones técnicas suministrada por el fabricante (SunPumps) se ha comprobado que ambas coinciden dentro de ciertos márgenes de error que son lógicos debido a la simplicidad del sistema de medición. En la 
toma de datos de caudales (tomados con un Rotámetro) se ha cometido un error dado que el rotámetro no es digital y dificultad la exactitud de la medición.

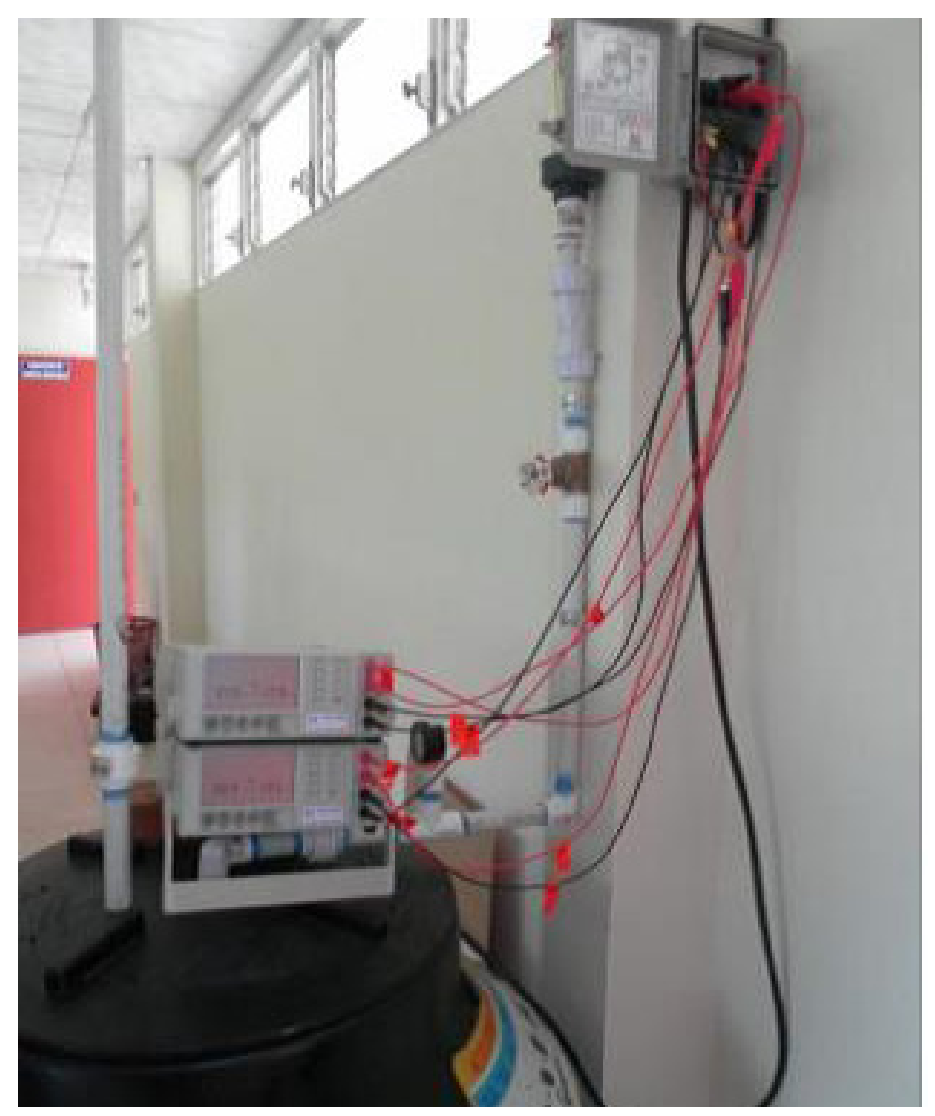

Con esta simplicidad del sistema de bombeo directo se considera que la experiencia ha sido un éxito, en cuanto a la defensa de la implantación de este tipo de equipos para países con recursos económicos reducidos, es sin duda, una solución óptima para aquellas comunidades de nuestro país que necesitan de unas cantidades de agua moderadas a diario (abastecimiento de agua potable).

\begin{tabular}{lll}
\hline $\begin{array}{c}\text { Potencia del panel } \\
(\mathrm{w})\end{array}$ & $\begin{array}{l}\text { Caudal } \\
(\text { lits } / \mathrm{m})\end{array}$ & \multicolumn{1}{c}{$\begin{array}{c}\text { Altura Manometrica } \\
(\mathrm{m})\end{array}$} \\
\hline 66 & 19 & 0 \\
71 & 18.5 & 3.5 \\
77 & 18 & 7 \\
91 & 17.5 & 10.5 \\
110 & 17.2 & 14 \\
128 & 17 & 17.5 \\
145 & 16.8 & 21 \\
176 & 16.7 & 24 \\
\hline
\end{tabular}

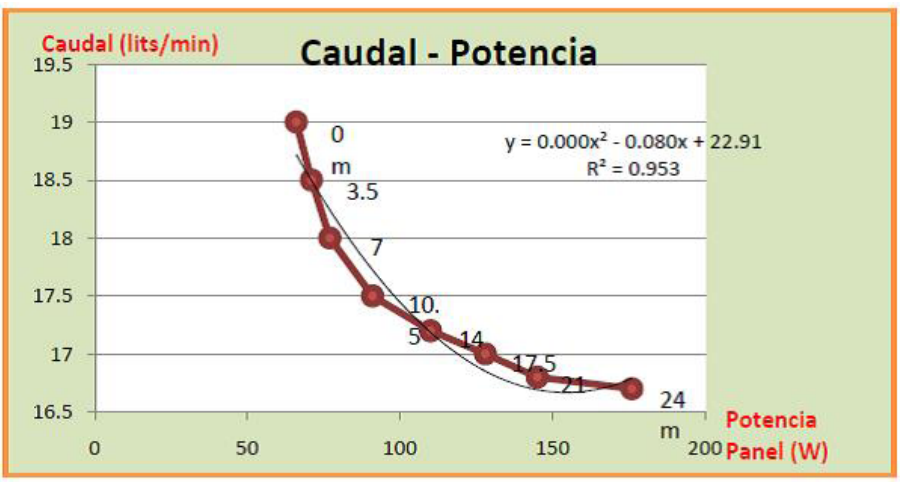

\begin{tabular}{llllccccc}
\hline Medida & PSI & $\begin{array}{c}\text { Altura } \\
\text { Manometrica (m) }\end{array}$ & $\begin{array}{c}\text { Irradiación } \\
\left(\mathrm{Wm}^{2}\right)\end{array}$ & $\begin{array}{c}\text { Tensión } \\
\text { Bomba }(\mathrm{V})\end{array}$ & $\begin{array}{c}\text { Intensidad } \\
\text { Bomba (A) }\end{array}$ & $\begin{array}{c}\text { Potencia } \\
\text { Motor } \\
\text { (Wats) }\end{array}$ & $\begin{array}{c}\text { Potencia } \\
\text { del panel }\end{array}$ & $\begin{array}{c}\text { Caudal } \\
(\text { lits/min) }\end{array}$ \\
\hline 1 & 0 & 0 & 1000 & 30.50 & 1.83 & 55 & 66 & 19.00 \\
2 & 2 & 3.5 & 1000 & 30.50 & 1.98 & 59 & 71 & 18.50 \\
3 & 10 & 7 & 1000 & 30.50 & 2.10 & 64 & 77 & 18.00 \\
4 & 15 & 10.5 & 1000 & 30.50 & 2.50 & 76 & 91 & 17.50 \\
5 & 20 & 14 & 1000 & 30.50 & 3.02 & 92 & 110 & 17.20 \\
6 & 25 & 17.5 & 1000 & 30.50 & 3.50 & 107 & 128 & 17.00 \\
7 & 30 & 21 & 1000 & 30.50 & 3.97 & 121 & 145 & 16.80 \\
8 & 35 & 24.6 & 1000 & 30.50 & 4.50 & 137 & 176 & 16.70 \\
1 & 0 & 0 & 500 & 30.40 & 1.99 & 61 & 73 & 18.00 \\
2 & 5 & 3.5 & 500 & 30.40 & 2.54 & 77 & 92 & 17.00 \\
3 & 10 & 7 & 500 & 29.60 & 2.80 & 83 & 100 & 16.00
\end{tabular}




\begin{tabular}{lllllllll}
4 & 15 & 10.5 & 500 & 27.00 & 3.03 & 82 & 98 & 15.00 \\
5 & 20 & 14 & 500 & 23.50 & 3.26 & 77 & 92 & 12.50 \\
6 & 25 & 17.5 & 500 & 22.70 & 3.54 & 80 & 96 & 11.30 \\
7 & 30 & 21 & 500 & 18.50 & 4.30 & 79 & 95 & 9.50 \\
8 & 35 & 24.6 & 500 & 18.10 & 4.66 & 84 & 101 & 9.10 \\
\hline
\end{tabular}

\section{Tabla 9: datos experimentales}

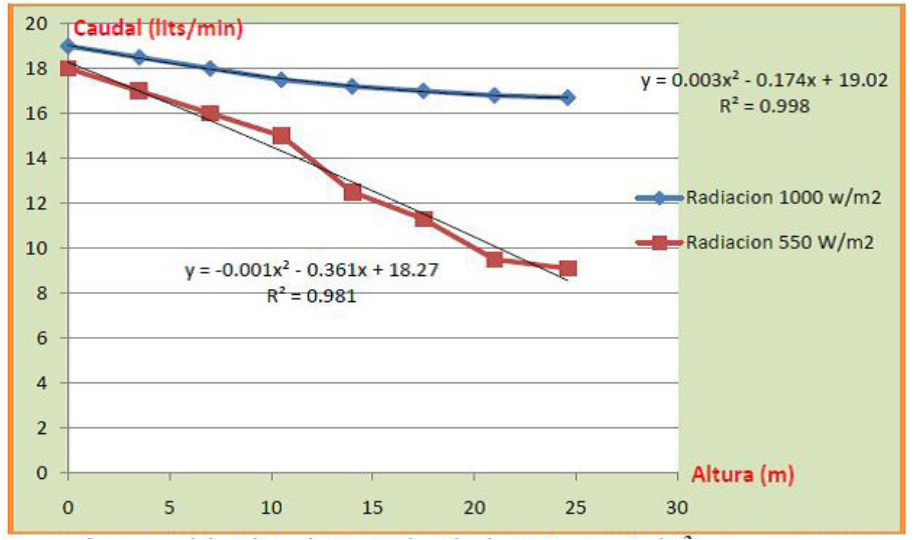

Gráfica 3: Caudal-Altura (Para irradiación de 1000 y $500 \mathrm{~W} / \mathrm{m}^{2}$ respectivamente)

\begin{tabular}{llll}
\hline Medición & $\begin{array}{l}\text { Irradiación } \\
\left(\mathrm{W} / \mathrm{m}^{2}\right)\end{array}$ & $\begin{array}{l}\text { Potencia de la } \\
\text { Bomba }(\mathrm{W})\end{array}$ & $\begin{array}{l}\text { Caudal } \\
(1 / \mathrm{min})\end{array}$ \\
\hline 1 & 150 & 21 & 2 \\
2 & 250 & 44 & 6 \\
3 & 350 & 49 & 8 \\
4 & 450 & 61 & 10 \\
5 & 550 & 76 & 12.5 \\
6 & 650 & 100 & 16 \\
7 & 750 & 102 & 16.2 \\
8 & 850 & 104 & 16.4 \\
9 & 950 & 105 & 16.7 \\
10 & 1000 & 106 & 17 \\
\hline
\end{tabular}

Tabla 11: Datos obtenidos para una altura manométrica de $14 \mathrm{~m}$ (altura del ensayo)
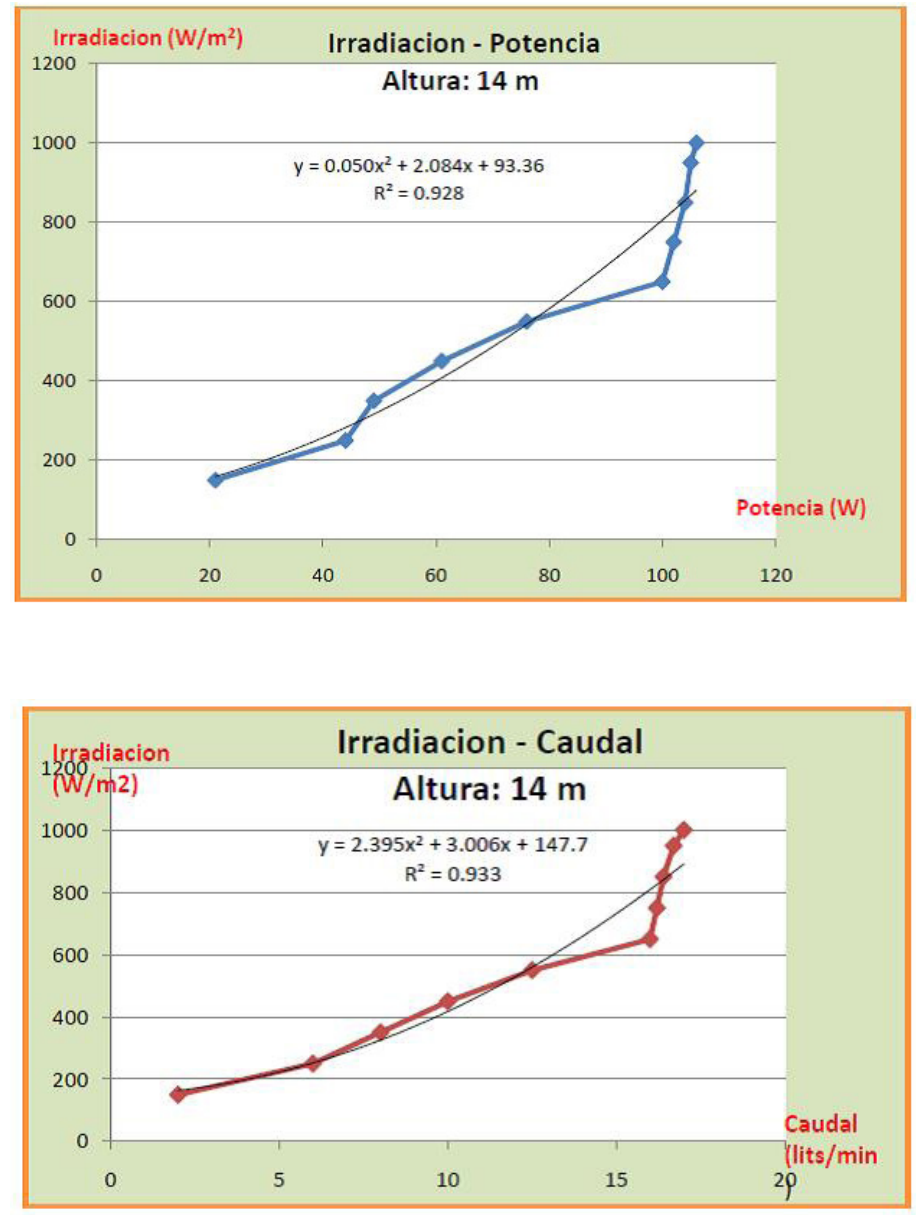


\section{CONCLUSIONES}

El prototipo fue dimensionado e instalado en el Laboratorio de Energías Renovables y se mostró como una herramienta didáctica confiable y práctica para determinar el comportamiento de sistemas de bombeo. Con la finalidad de caracterizar el prototipo, se usaron instrumentos de medición que posibilitaron la determinación de las características eléctricas del generador (panel fotovoltaico) y las características hidráulicas de la bomba. Se monitoreó el sistema instalado a fin de precisar el máximo y mínimo caudal posible, según los niveles de irradiáncia proporcionados por los módulos fotovoltaicos.

Se realizó una serie de ensayos con bombeo directo (panel solar) y Baterías, con la bomba accionadas con motor de corriente continua de $24 \mathrm{~V}$ y baja potencia, que permitió la caracterización del sistema y el comportamiento del conjunto motor-bomba. El comportamiento presentado por la bomba resultó ser el esperado, según lo especificado por la bibliografía especializada y las características informadas por el fabricante de la bomba.

Existió cierta dificultad para la validación del prototipo (captura de datos de la radiación), pero aun así se determinaron las máximas variaciones de caudal, altura, potencia e irradiación.

En relación a la hipótesis de esta investigación podemos decir que se ha cumplido, pues los resultados del experimento han brindado los datos que permiten asegurar el cumplimiento y sentenciar con toda seguridad que, "En los sistemas de bombeo fotovoltaico, el caudal de agua y la potencia de la bomba, es directamente proporcional a la radiación solar incidente en el plano del generador fotovoltaico, e inversamente proporcional a la altura manométrica". Es importante mencionar que el prototipo está cumpliendo con su principal objetivo que es de ser utilizado como medio didáctico, puesto que ya se está entrenando estudiantes en el estudio de estos sistemas, manipulando el sistema y enseñándoles a la toma de datos de los principales parámetros de medición y dimensionado.

\section{RECOMENDACIONES}

En relación a la utilización de este prototipo como medio didáctico, se consideran importantes las siguientes recomendaciones:

1. El docente deberá tener conocimientos sobre los sistemas de bombeo accionados con energía solar.

2. Realizar un guion de laboratorio, en el que se incluya de forma detallada todo el procedimiento de la práctica a realizar.

3. Tomar en cuenta las medidas de seguridad, para evitar accidentes en el laboratorio.

4. Se debe promover el aprendizaje básico, sobre las energías renovables, de forma manipulativa para conseguir un aprendizaje más significativo y duradero.

5. Utilizar este prototipo, para realizar mediciones en configuraciones distintas, es decir ensayos con baterías y con paneles solares.

6. Hacer mejoras en el prototipo siempre que, se consideren necesarios, esto permitirá entrenar estudiantes y profesionales en este campo.

\section{BIBLIOGRAFÍA}

Abella, M, (2005) "Sistemas Fotovoltaicos: Introducción al diseño y dimensionado de instalaciones de energía solar fotovoltaica". 2 ed

Arnalich, Santiago. (2008). "La instalación de un sondeo" I ed.

Cervantes, J. (2009). Estudio de Sistemas de Bombeo. Cedenit. Cuernavaca, Morelos, México.

Gasquet, H. (2004). Conversión de la Luz Solar en Energía Eléctrico. Manual teórico y práctico sobre los sistemas Fotovoltaicos. Cuernavaca, Morelos.

Graham, Barrientos, Briones, Foster, Ascencio \& Arango (2001). “Guía para el desarrollo 
de proyectos de bombeo de agua con energía fotovoltaica". Sandia National Laboratories.

Miranda, Villegas, Buitrago, Barrau, Gonzales, Vargas \& Alcázar (2009). Energía Solar Fotovoltaica, 3a Ed.

SDS Series Pumps and PCA Series Pump Controllers. www.sunpumps.com. 10 de julio de 2012
Vera, Steigleder, Aeberhard, \& Benítez (2006). Bancada de Ensayo para Determinar el Desempeño de SBFV de Baja Potencia. Universidad Nacional de Nordeste, Porto Alegre, Rio Grande do Sul, Brasil.

Vilora, J. Motores eléctricos. Accionamiento de Máquinas. 30 Tipos de Motores. $4^{\mathrm{a}}$ edición. Thomson . p. 110. 